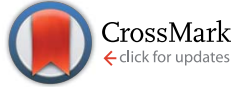

Cite this: RSC Adv., 2016, 6, 37195

Received 20th February 2016

Accepted 5th April 2016

DOI: $10.1039 / c 6 r a 04595 b$

www.rsc.org/advances

\section{Microheterogeneity in binary mixtures of methanol with aliphatic alcohols: ATR-IR/NIR spectroscopic, chemometrics and DFT studies}

\author{
Władysław Wrzeszcz, Paweł Tomza, Michał Kwaśniewicz, Sylwester Mazurek, \\ Roman Szostak and Mirosław Antoni Czarnecki*
}

\begin{abstract}
This paper reports ATR-IR and NIR spectroscopic studies on microheterogeneity in binary mixtures of methanol with five short chain aliphatic alcohols: ethanol, 1-propanol, 2-propanol, tert-butanol and cyclopentanol. The inhomogeneity distribution of molecules in the mixtures was characterized by MIR/ NIR excess absorption spectra, two-dimensional correlation approach, and chemometric methods. The experimental data were compared and discussed with the theoretical calculations of homo- and heteroclusters of different size and composition. The results obtained in the present work allow us to infer that all studied mixtures deviate from the ideal and the largest effect appears at equimolar mixture. At this composition about $50 \%$ of molecules are involved in the mixed clusters. In the entire range of compositions in the mixtures coexist the clusters of pure alcohols and heteroclusters with an average mole ratio of $1: 1$. Only in methanol/tert-butanol mixture was observed an additional tert-butanol-rich cluster with an average methanol : tert-butanol mole ratio between $1: 3$ and $1: 4$. The excess absorption and two-dimensional correlation spectra reveals that methanol/ethanol mixture is the closest to the ideal one, while the largest degree of non-ideality was observed for methanol/tert-butanol mixture. The extent of deviation from the ideal mixture increases with an increase in the chain length and the order of the alcohol. These trends are correlated with decreasing degree of self-association of the alcohol.
\end{abstract}

\section{Introduction}

It is well known that alcohol-water mixtures reveal non-ideal behavior due to the presence of hydrophobic interactions disturbing the structure of liquid water..$^{1-6}$ As a result, the molecules of water and alcohol form separate clusters predominantly composed of one constituent of the mixture. This behavior is not surprising since, in contrast to the molecules of alcohol, the molecules of water do not posses the hydrophobic part. Inhomogeneous distribution was also found in the binary mixture of methanol with acetone. ${ }^{-9}$ Musso et al. reported the presence of microscopic inhomogeneities in this mixture through the Raman noncoincidence effect. ${ }^{7}$ The authors speculate that this effect originates from hydrogen-bonding between molecules of methanol. Analysis of vibrational and NMR spectra of methanol/acetone mixture led Idrissi et al. to the conclusion that the molecules of acetone are not homogeneously distributed between the acetone mole fraction from 0.05 to $0.55 .^{8}$ At higher mole fraction of acetone (from 0.55 to 1 ) the molecules of methanol are inhomogeneously distributed, but the extent of this effect is smaller than that for acetone. Theoretical calculations by Kollipost et al. have shown that the heterodimers and

Faculty of Chemistry, University of Wroctaw, F. Joliot-Curie 14, 50-383 Wrocław, Poland. E-mail: miroslaw.czarnecki@chem.uni.wroc.pl; Fax: +48-71-3282348 heterotrimers of methanol and acetone are more favorable than the corresponding homoclusters. ${ }^{9}$ In contrast, pure tetramers and higher homoclusters are more stable than the mixed clusters. Tan et al. have demonstrated that the relative terahertz absorption coefficients of the diol/methanol binary mixtures are close to zero. ${ }^{10}$ On this basis the authors concluded that these mixtures can be considered ideal.

When two solvents with similar structure and properties are mixed with each other, one can expect behavior close to the ideal. Ortega et al. suggested that this behavior should be observed for the mixtures which do not present preferential solvation. ${ }^{\mathbf{1 1}}$ The composition dependence of the solvatochromic parameters for mixtures of methanol with the most similar alcohols (including ethanol) suggests ideal behavior. ${ }^{11}$ Thus, it was surprising when Mello et al. reported the microstructures formation in a seemingly ideal homogeneous mixture of methanol and ethanol. ${ }^{12}$ The authors claim that methanol and ethanol molecules form separate clusters at a molecular level and the distinct clusters interact weakly through cohesion or dispersion forces. For the first time it was reported heterogeneity at a molecular level in a non-aqueous mixture of very similar compounds. So far this is the only report on microheterogeneity in binary mixture of methanol and ethanol, but Mello et al. speculate that analogous phenomenon should be observed in other mixtures of alcohols. ${ }^{12}$ 
Inspired by this work we undertook more detailed and comprehensive studies of the microheterogeneity in binary mixtures of alcohols. The first purpose of this study is to verify the finding by Mello et al. ${ }^{12}$ Next, we examine if this effect occurs for other mixtures of alcohols. Finally, we elucidate the relationship between the molecular structure of the alcohol and the extent of the microheterogeneity in binary mixtures of alcohols. To realize these aims we performed ATR-IR and NIR spectroscopic studies on binary mixtures of methanol with five aliphatic alcohols of different size and structure of the chain. The size of these alcohols was relatively small to enable theoretical modeling of larger clusters. The inhomogeneity distribution of molecules in the mixtures was characterized by MIR/ NIR excess absorption spectra, ${ }^{\mathbf{1 3 , 1 4}}$ two-dimensional (2D) correlation analysis, ${ }^{15-17}$ and chemometric methods like principal component analysis (PCA), ${ }^{18}$ evolving factor analysis (EFA) ${ }^{19}$ and multivariate curve resolution (MCR). ${ }^{20,21}$ In particular, the excess absorption spectra and 2D correlation analysis are very sensitive tools in detection of small deviations from the linearity. Besides, we proposed semi-quantitative parameters related to the degree of deviation from the ideality. These parameters were calculated from the MIR/NIR excess absorption and 2D correlation spectra. The experimental results were compared and discussed with the theoretical calculations (DFT) of numerous model structures including homo- and heteroclusters of different size and composition.

\section{Experimental}

We studied binary mixtures of methanol (ME) with five aliphatic alcohols: ethanol (ET), 1-propanol (1PA), 2-propanol (2PA), tertbutanol (TB) and cyclopentanol (CP). Alcohols of high purity (>99\%) were purchased from Aldrich Chemical Co. (Germany), J. T. Baker (The Netherlands), Fluka (Germany) and $\mathrm{POCH}$ (Poland). All samples were distilled and dried under freshly activated molecular sieves (4A). ATR-IR and FT-NIR spectra were recorded at a resolution of $2 \mathrm{~cm}^{-1}$ and $4 \mathrm{~cm}^{-1}$ (256 scans), respectively, on Nicolet Magna 860 spectrometer with DTGS detector. ATR-IR spectra were recorded with multi-reflection ZnSe crystal (PIKE), while NIR spectra were measured in a variable temperature quartz cell (Hellma) of $5 \mathrm{~mm}$ thickness. The spectral measurements were performed at $25{ }^{\circ} \mathrm{C}$, only $\mathrm{ME} / \mathrm{TB}$ mixture was measured at $30{ }^{\circ} \mathrm{C}$ (melting point of $\mathrm{TB}$ is $\approx 26$ ${ }^{\circ} \mathrm{C}$ ). The spectra of all mixtures were recorded in the entire range of mole fractions $(X)$ with a step of $0.04 \pm 0.0001$. The samples were automatically prepared and dosed to ATR-IR and NIR cells by a special flow-system designed and developed in our laboratory. This way, the spectral measurements were more precise and reliable. These features are especially important in detection of small fluctuations of spectral intensity.

\section{Data analysis}

The baseline fluctuations were minimized by an offset at 4000 $\mathrm{cm}^{-1}$ (ATR-IR) or $9000 \mathrm{~cm}^{-1}$ (NIR), and no other corrections were made on the spectra. The generalized 2D correlation spectra were calculated according to Noda's algorithm, ${ }^{\mathbf{1 5 , 1 6}}$ using Matlab 7.0.4 (The Math Works Inc.) based software ${ }^{\mathbf{1 7}}$ and an average spectrum was used as a reference. The asynchronous intensity was multiplied (array multiplication) by corresponding synchronous intensity. The rules for interpretation of $2 \mathrm{D}$ correlation spectra were reported elsewhere. ${ }^{15-17}$ The number of species present in the data set and the initial estimates of the concentration profiles for each of the component were obtained from PCA and EFA. ${ }^{18,19}$ The actual concentration and spectral profiles of the pure components were resolved by multivariate curve resolution-alternating least squares (MCR-ALS) approach with constraints (non-negativity on concentrations and spectra, closure, equality). ${ }^{20,21}$ The chemometric analysis was performed by PLS-Toolbox 6.2 (Eigenvector Research Inc.) for use with Matlab.

The spectrum of the ideal binary mixture $\left(A_{\text {id }}\right)$ was calculated as follows:

$$
A_{\text {id }}=A_{1} \times X_{1}+A_{2} \times\left(1-X_{1}\right)
$$

where $A_{1}$ and $A_{2}$ are the MIR/NIR spectra of neat alcohols and $X_{1}$ is the mole fraction corresponding to $A_{1}$. Next, we calculated the excess MIR/NIR absorption spectra $\left(A_{\text {exc }}\right)$ as a difference between the real spectra $(A)$ and the $A_{\text {id }}$ spectra:

$$
A_{\text {exc }}=A-A_{\text {id }}
$$

The excess spectrum reveals deviations of the mixture from the ideality. If the mixture is close to the ideal one, the intensities in the excess spectrum are near to zero, while appearance of significant positive or negative values indicates the non-ideal behavior.

\section{Theoretical calculations}

DFT calculations were performed at the B3LYP/6-311++G(d,p) level of theory using Gaussian $03 \mathrm{~W} .{ }^{22}$ The total energy of each structure was corrected with the zero point vibrational energy, and the harmonic frequencies were calculated. All stationary points were found to be true minima since no imaginary frequencies were obtained. The DFT calculations were performed in WCSS (Wrocław, grant 163).

\section{Results and discussion}

\section{ATR-IR and NIR spectra}

ATR-IR and NIR spectra were recorded from 1000 to $4000 \mathrm{~cm}^{-1}$ and from 4000 to $9000 \mathrm{~cm}^{-1}$, respectively. However, for analysis we selected the most important ranges including the fundamentals of the $\mathrm{CH} / \mathrm{OH}$ stretching vibrations $\left(2700-3800 \mathrm{~cm}^{-1}\right)$ and the corresponding overtones (6000-9000 $\mathrm{cm}^{-1}$ ). In Fig. 1 and 2 are shown exemplary MIR and NIR spectra of ME/ET and $\mathrm{ME} / \mathrm{TB}$ mixtures in the entire range of mole fractions. The broad spectral feature absorbing from 3100 to $3600 \mathrm{~cm}^{-1}$ was assigned to the hydrogen-bonded $\mathrm{OH}$, while its first overtone appears in the $6200-7000 \mathrm{~cm}^{-1}$ range. Interestingly, the band due to the free $\mathrm{OH}$ is not visible in the fundamentals region. This band is hardly seen near $3615 \mathrm{~cm}^{-1}$ in the second derivative spectra (not shown). In contrast, the free $\mathrm{OH}$ clearly appears in the NIR 

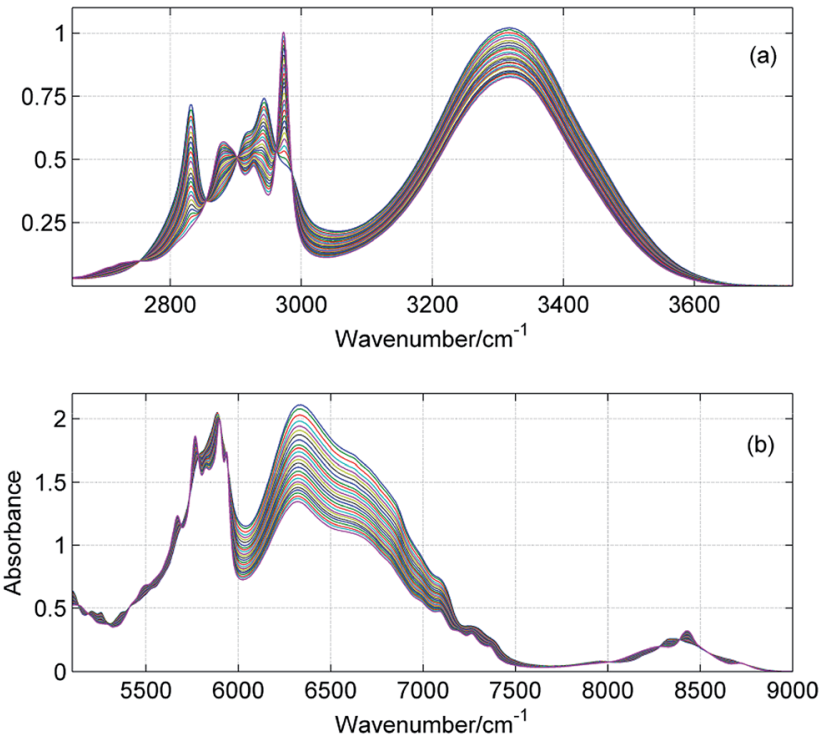

Fig. 1 ATR-IR (a) and NIR (b) spectra of ME/ET mixture in the entire range of mole fractions with a step of 0.04 .
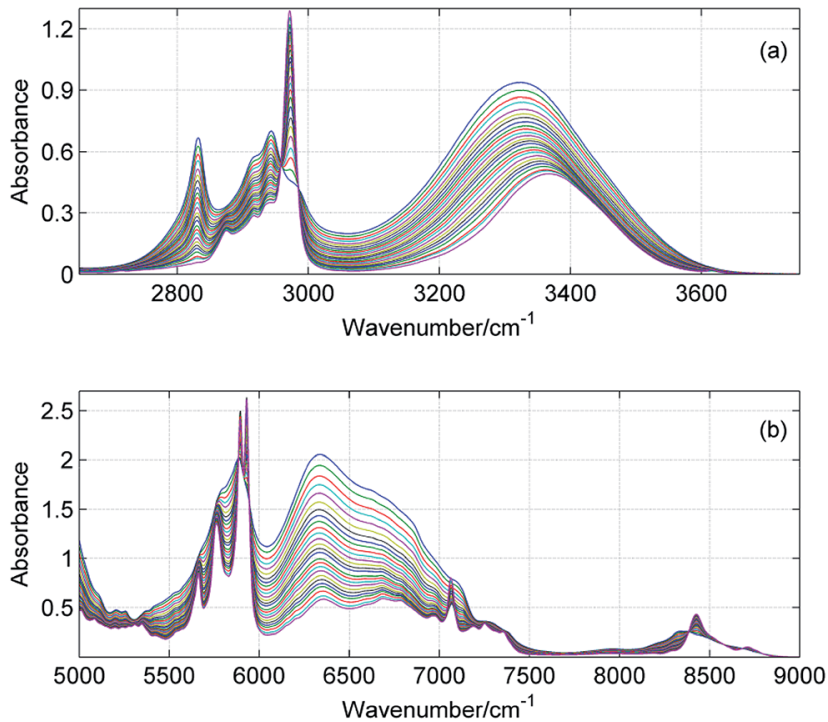

Fig. 2 ATR-IR (a) and NIR (b) spectra of ME/TB mixture in the entire range of mole fractions with a step of 0.04 .

spectra near $7100 \mathrm{~cm}^{-1} \cdot{ }^{23}$ From 2800 to $3000 \mathrm{~cm}^{-1}$ absorbs the bands due to the $\mathrm{CH}$ stretching vibrations, while the first overtones absorb from 5750 to $6000 \mathrm{~cm}^{-1}$. In a few mixtures these bands were too intense $(A>2)$ and hence, this spectral range was excluded from the analysis. Instead, we applied the second overtone of the $\mathrm{CH}$ stretching vibrations which is much weaker and absorb from 8200 to $8800 \mathrm{~cm}^{-1}$.

Fig. 3 displays selected spectral changes as a function of the mixture composition. It is of note that the intensity of the bonded $\mathrm{OH}$ continuously decreases, while position of this band does not change up to $X_{\mathrm{TB}}=0.4-0.5$. When $X_{\mathrm{TB}}$ still further increases this band shifts to higher wavenumbers. From Fig. 3c
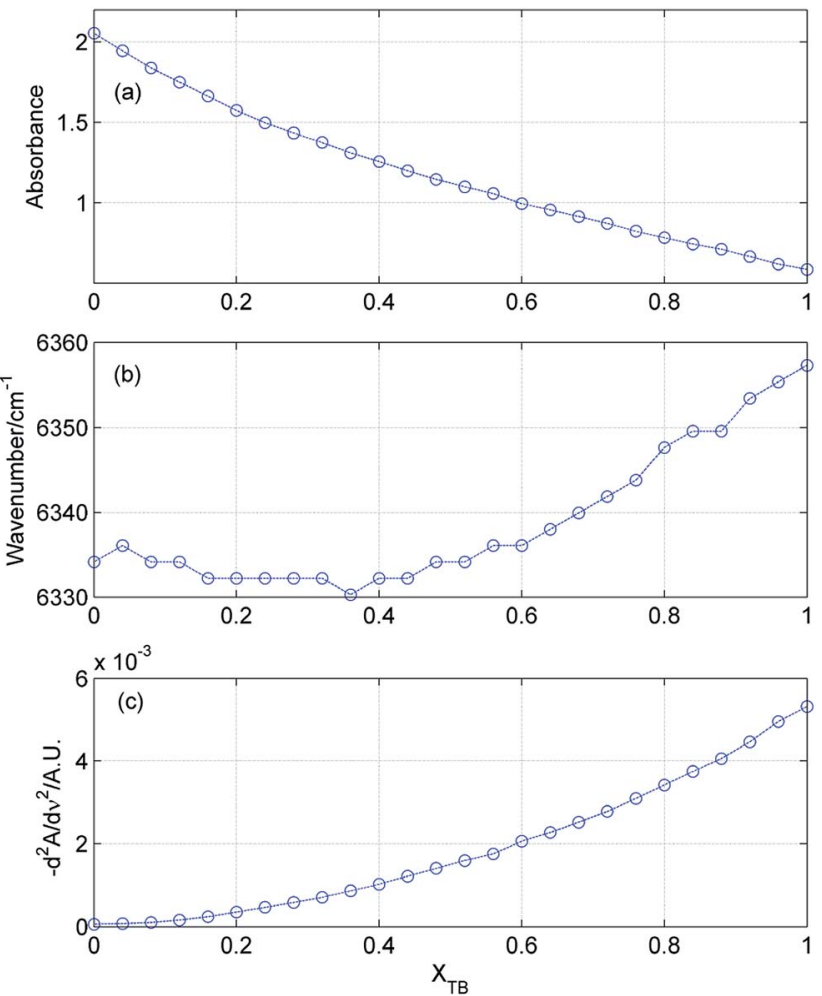

Fig. 3 Absorbance (a) and position (b) of the bonded $\mathrm{OH}$ in ME/TB mixture as a function of TB mole fraction. In (c) is shown absorbance (in second derivative spectrum) of the free $\mathrm{OH}$ from TB (at $7067 \mathrm{~cm}^{-1}$ ).

it results that the population of the free $\mathrm{OH}$ groups from $\mathrm{TB}$ increases with the increase in $X_{\mathrm{TB}}$. However, this increase is not a linear function of the composition of the mixture. All of these observations demonstrate a complex character of the spectral changes in the mixture.

\section{Estimation of the number of components in the mixture}

Estimation of the number of distinct species present in the mixture is the first step of the chemometric analysis. In Table 1 are summarized results of PCA, EFA and 2D correlation analysis for all studied mixtures. In most cases the chemometric analysis does not provide a clear-cut answer on the number of components and indicates the presence of two or three components. Only for $\mathrm{ME} / \mathrm{TB}$ mixture the analysis suggests the existence of four (EFA) or five (PCA/ATR-IR) different species. As mentioned

Table 1 Number of components from PCA, EFA, 2D correlation analysis and MCR-ALS for all studied mixtures

\begin{tabular}{|c|c|c|c|c|c|c|c|c|}
\hline \multirow[b]{2}{*}{ Mixture } & \multicolumn{4}{|c|}{ ATR-IR } & \multicolumn{4}{|l|}{ NIR } \\
\hline & PCA & EFA & 2DCOS & MCR & PCA & EFA & 2DCOS & MCR \\
\hline ME/ET & 2 & 2 & 3 & 3 & 2 & 3 & 3 & 3 \\
\hline ME/1PA & 3 & 3 & 3 & 3 & 2 & 3 & 3 & 3 \\
\hline $\mathrm{ME} / 2 \mathrm{PA}$ & 2 & 3 & 3 & 3 & 3 & 3 & 3 & 3 \\
\hline $\mathrm{ME} / \mathrm{CP}$ & 2 & 3 & 3 & 3 & 2 & 3 & 3 & 3 \\
\hline $\mathrm{ME} / \mathrm{TB}$ & 5 & 4 & 3 & 4 & 3 & 4 & 3 & 3 \\
\hline
\end{tabular}


before, 2D correlation analysis is a powerful tool for the resolution enhancement. ${ }^{15-17}$ The peaks are resolved in the asynchronous spectrum if the perturbation-dependent pattern of spectral changes for the component peaks is different. This suggests that the resolved peaks originate from different molecules or from the same molecule in unlike environments. Fig. 4 shows 2D ATR-IR asynchronous spectra of ME/ET mixture. Obviously, the peaks due to both the methyl (2827/ 2838 ) and methylene (2970/2977) groups are resolved into two components. This splitting appears in the asynchronous spectra of all remaining mixtures (not shown). Similar spectral pattern was observed by Mello et al. in the 2D Raman asynchronous spectra of ME/ET mixture. ${ }^{12}$ From this splitting the authors assumed the presence of separate ME and ET clusters without hydrogen bonding between different alcohol species. It is of note that they excluded the presence of heteroclusters in the mixture. However, we interpret this splitting in a different way. The asynchronous spectra develop a pair of peaks for both alcohols. One peak of each pair can be assigned to the pure component, while the other peak originates from the mixed cluster. The pattern of hetero-peaks between the methyl and methylene groups (Fig. 4) does not reveal additional components. Hence, the double asynchronous peaks for both components of the mixture indicate the presence of three distinct species: two kinds of homoclusters and one kind of heterocluster.

\section{Resolution of the concentration and spectral profiles}

Fig. 5 and 6 display the concentration and spectral profiles obtained from MCR-ALS of ATR-IR and NIR spectra of ME/ET mixture by using three components. One of the concentration profiles decreases with the increase in $X_{\mathrm{ET}}$, while the other profile reveals an opposite behavior. Hence, it seems reasonable

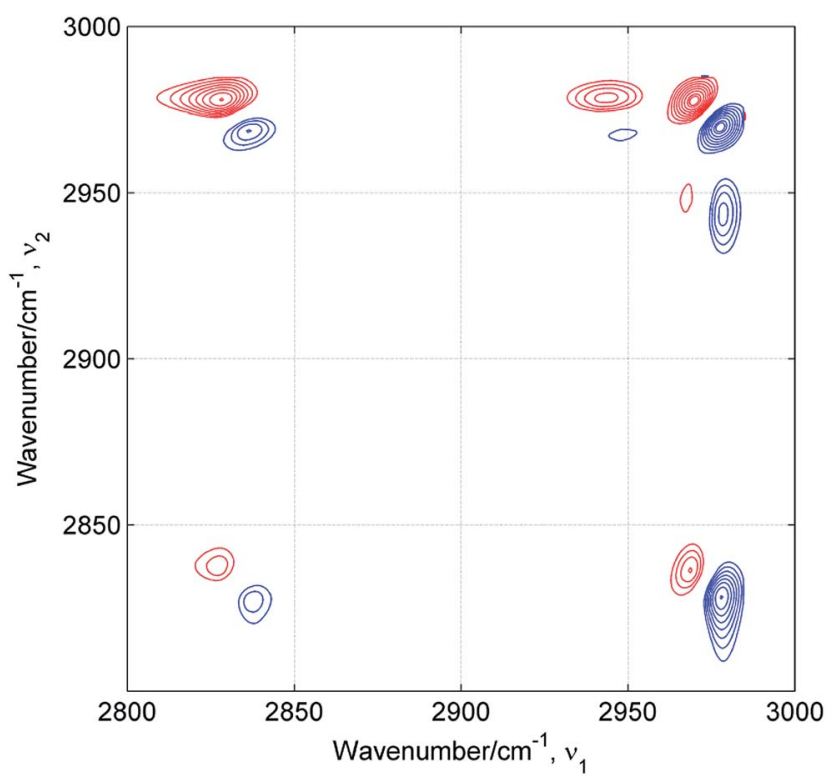

Fig. 4 2D ATR-IR asynchronous spectra of ME/ET mixture. Positive and negative peaks are drown in red and blue, respectively.
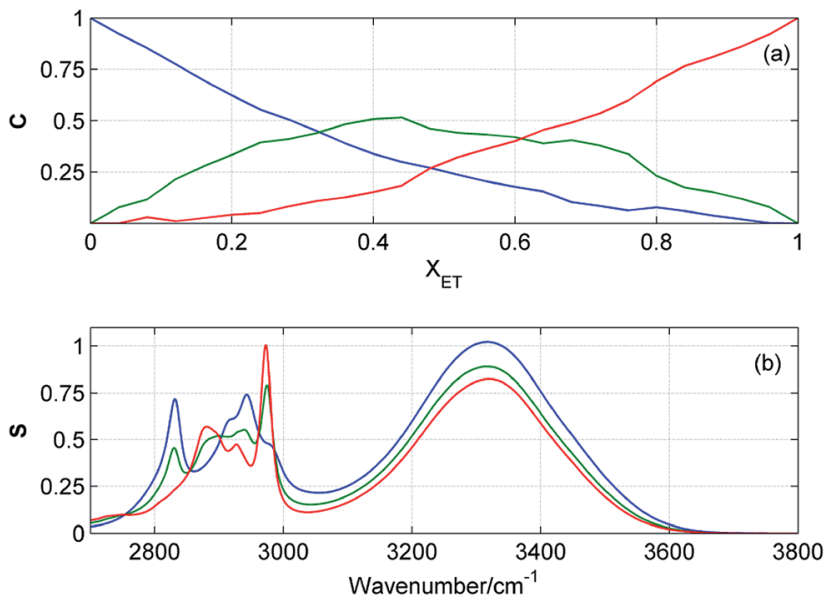

Fig. 5 Concentration (a) and spectral (b) profiles obtained from MCRALS of ATR-IR spectra of ME/ET mixture by using three components. Corresponding concentration and spectral profiles have the same colors.
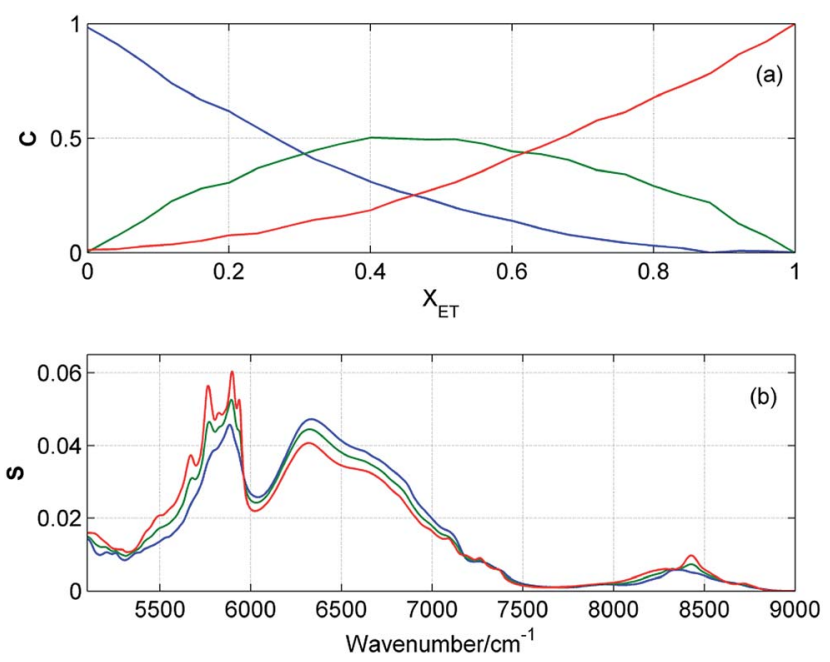

Fig. 6 Concentration (a) and spectral (b) profiles obtained from MCRALS of NIR spectra of ME/ET mixture by using three components. Corresponding concentration and spectral profiles have the same colors.

to assign these profiles to pure ME and ET, respectively. In Fig. 7 and 8 are compared ATR-IR and NIR spectral profiles of ME and ET with the normalized spectra of bulk ME and ET. A striking similarity between the spectral profiles and the corresponding spectra of bulk alcohols confirms above assignment. Hence, these spectral profiles were assigned to ME and ET contributing to the homoclusters similar to those existing in bulk alcohols. On the other hand, the third component is completely different from the two others and has a maximum near $X_{\mathrm{ET}} \approx 0.5$ (Fig. 5a and $6 \mathrm{a}$ ). Of particular note is that the spectral profile of this component includes the peaks both from ME and ET (Fig. 5b and 6b). Evidently, this component originates from the heteroclusters, and its composition depends on the actual composition of the mixture. Assuming that the spectral profile of the 


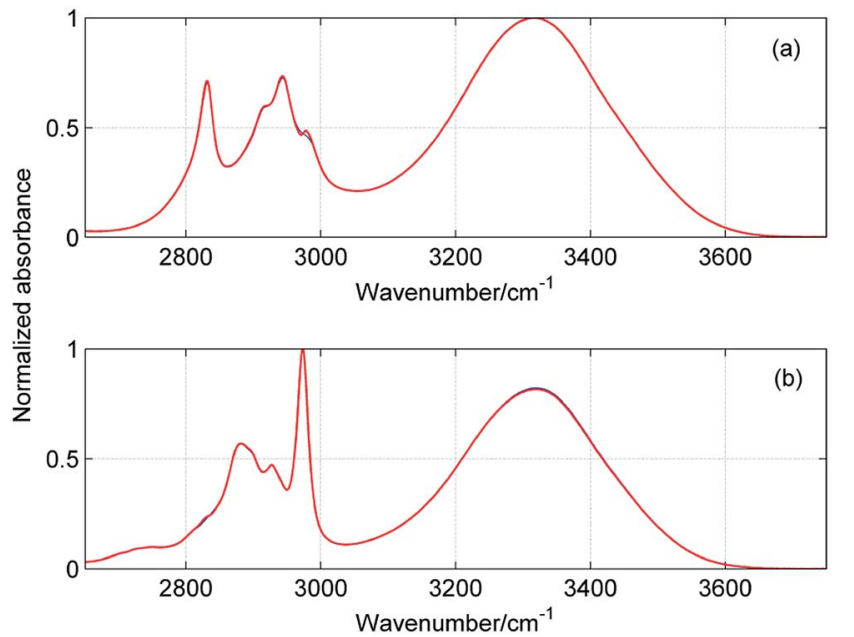

Fig. 7 Comparison of spectral profiles (blue) obtained from MCR-ALS of ATR-IR spectra of ME/ET mixture with the spectra (red) of pure methanol (a) and ethanol (b).

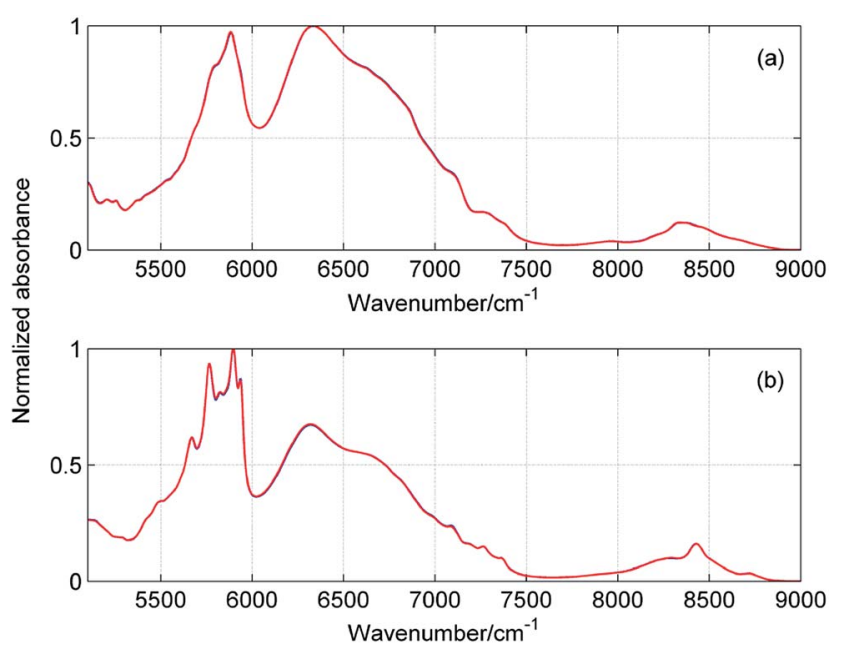

Fig. 8 Comparison of spectral profiles (blue) obtained from MCR-ALS of NIR spectra of ME/ET mixture with the spectra (red) of pure methanol (a) and ethanol (b).

mixed cluster can be represented as a linear combination of the spectra of pure alcohols (eqn (1)) one can estimate an average contribution from each alcohol to the mixed cluster. By solving eqn (1) we obtained $X_{\mathrm{ME}}=0.47$ and $X_{\mathrm{ET}}=0.53$ from ATR-IR spectra, and $\mathrm{X}_{\mathrm{ME}}=X_{\mathrm{ET}}=0.50$ from NIR spectra. This means that the mixed clusters have an average ME : ET molar ratio of $1: 1$. From Fig. $5 a$ and $6 a$ it is clear that at equimolar mixture nearly $50 \%$ molecules of ME and ET form the mixed clusters, while the remaining molecules are involved in the homoclusters. Similar results of MCR-ALS (not shown) were obtained for $\mathrm{ME} / 1 \mathrm{PR}, \mathrm{ME} / 2 \mathrm{PR}$ and $\mathrm{ME} / \mathrm{CP}$ mixtures.

Results of PCA and EFA suggest that in ME/TB mixture exist four or five different kinds of clusters. In spite of many efforts, we were not able to obtain reasonable results of MCR-ALS by using five components. Fig. 9 displays the concentration and spectral profiles of ME/TB mixture from MCR-ALS by using four components. Two of these profiles are assigned to the homoclusters of ME and $\mathrm{TB}$, while the two other profiles originate from the mixed clusters. Solving eqn (1) we estimated an average composition of both heteroclusters. One of these clusters has an average ME : TB molar ratio of $1: 1$, whereas for the second cluster this ratio is between $1: 3$ and $1: 4$. The highest population of both heteroclusters reaches $25 \%$ and for TB-rich cluster this maximum is shifted to higher $X_{\mathrm{TB}}$. By adding the contributions from these two mixed clusters we obtained the concentration profile (Fig. 9a, dotted brown line) similar to those shown in Fig. 5a and 6a. Like for the other mixtures, at equimolar composition about $50 \%$ of molecules are involved in the heteroclusters.

\section{Excess absorption spectra}

Fig. 10 shows the composition-mean excess ATR-IR and NIR absorption spectra for all studied mixtures. In both spectral ranges the magnitude of the changes is the smallest for ME/ET mixture, while the largest variations are observed for $\mathrm{ME} / \mathrm{TB}$ mixture. To compare more quantitatively the results obtained for different mixtures, we calculated the value of ER defined as below:

$$
\mathrm{ER}=\frac{\int \mid \text { mean }(\mathrm{MIR} / \mathrm{NIR} \text { excess spectrum }) \mid}{\int \text { mean }(\mathrm{MIR} / \mathrm{NIR} \text { spectrum })} \times 100 \%
$$

where 'mean' stands for the composition-mean MIR/NIR excess or normal spectrum. For an ideal mixture ER is expected to be close to zero and this value should significantly depart from zero for non-ideal mixtures. In Table 2 are collected values of ER for all studied mixtures. It is of note that all ER are markedly
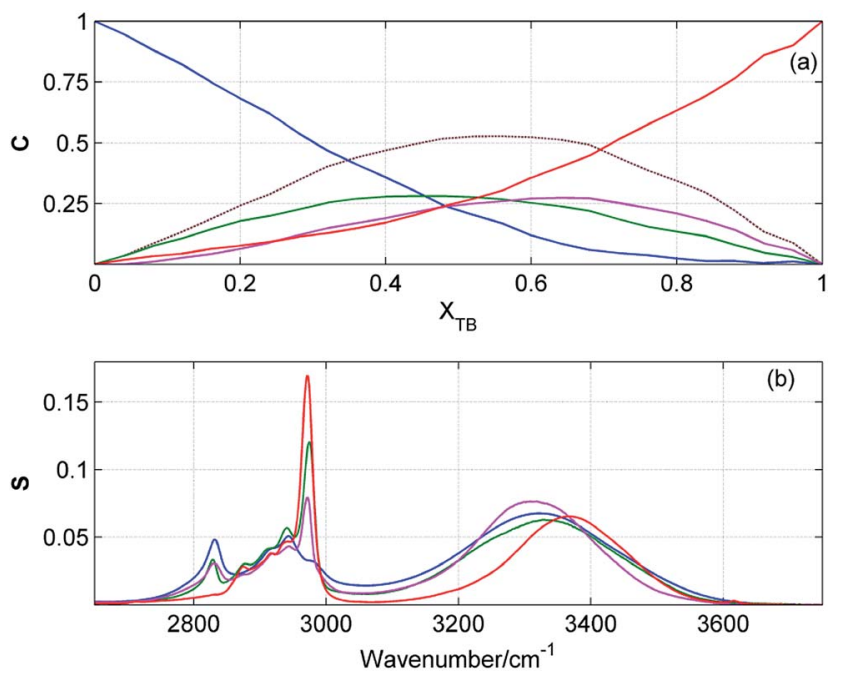

Fig. 9 Concentration (a) and spectral (b) profiles obtained from MCRALS of ATR-IR spectra of ME/TB mixture by using four components. Corresponding concentration and spectral profiles have the same colors. Concentration profile drawn by dashed brawn line is a sum of the green and magenta profiles. 

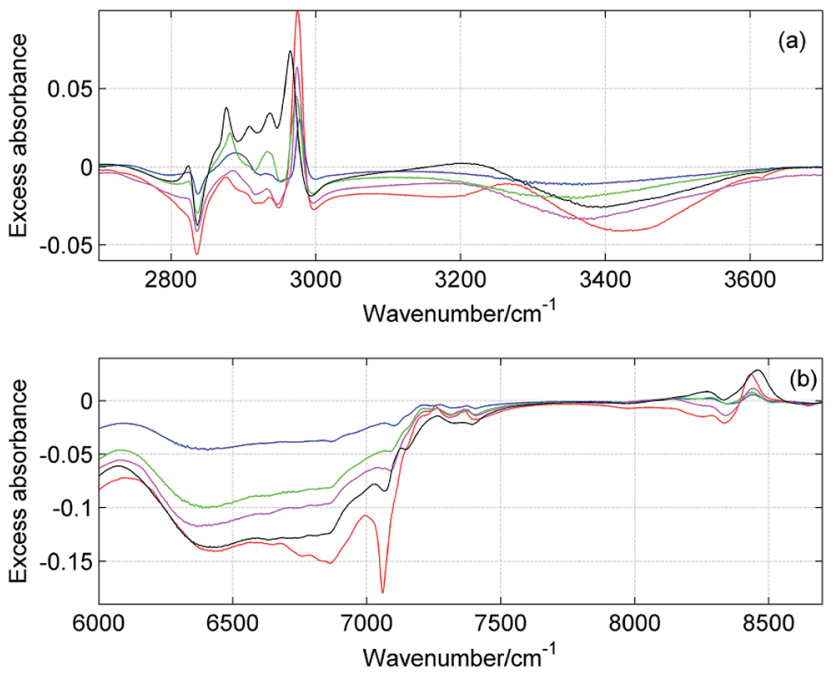

Fig. 10 Composition-mean excess MIR (a) and NIR (b) absorption spectra of ME/ET (blue), ME/1PA (green), ME/2PA (magenta), ME/CP (black) and ME/TB (red) mixtures.

Table 2 Parameters of ER and DA for all studied mixtures

\begin{tabular}{lllllr}
\hline & ATR-IR & & & NIR & \\
\cline { 2 - 3 } \cline { 5 - 6 } Mixture & ER & DA & & ER & DA \\
\hline ME/ET & 1.5 & 4.9 & & 2.7 & 2.3 \\
ME/1PA & 2.9 & 6.8 & 6.5 & 3.9 \\
ME/2PA & 2.6 & 7.2 & & 8.2 & 5.9 \\
ME/CP & 3.5 & 7.6 & & 10.5 & 7.2 \\
ME/TB & 7.6 & 8.7 & 12.9 & 20.1
\end{tabular}

different from zero. The smallest ER was obtained for ME/ET mixture, while the largest one for $\mathrm{ME} / \mathrm{TB}$ mixture. This suggests that behavior of ME/ET mixture is the closest to the ideal mixture. It is not surprising since these two alcohols have the most similar structures and properties. Interestingly, ME/TB mixture deviates from the ideality more than ME/CP mixture. Among two isomers of propyl alcohol, mixture of ME with the primary alcohol is more ideal. These results allow us to conclude that the degree of non-ideality increases upon an increase in the chain length and the order of the alcohol. It is of note that this trend is correlated with the degree of selfassociation of the alcohol. ${ }^{23}$ Hence, one can conclude that more associated alcohols from more ideal binary mixtures with ME. This observation is in line with the statement of Tan et al. that $\mathrm{ME} /$ diol mixtures can be considered ideal. ${ }^{10}$ NIR spectroscopic studies reveal that the aliphatic diols are more associated than the aliphatic alcohols. ${ }^{17,23}$

Based on 2D correlation spectra we proposed another parameter related to the amount of nonlinearity of spectral changes. The degree of asynchronicity (DA) was defined as:

$$
\mathrm{DA}=\frac{\operatorname{asyn}_{\max }-\operatorname{asyn}_{\min }}{\operatorname{syn}_{\max }-\operatorname{syn}_{\min }} \times 100 \%
$$

where min and max refer to minimum and maximum intensity at the synchronous (syn) and asynchronous (asyn) spectrum. Higher value of DA indicates the higher non-linearity of spectral changes. An inspection of Table 2 reveals that this value is well correlated with ER. Again, the smallest DA was obtained for ME/ ET mixture, while the largest DA was found for $\mathrm{ME} / \mathrm{TB}$ mixture.

In order to estimate the composition of the mixtures with the largest deviations from the ideality we examined the excess absorption spectra in detail. In Fig. 11 and 12 are displayed the intensity changes for selected MIR and NIR bands in the excess absorption spectra of $\mathrm{ME} / \mathrm{ET}$ and $\mathrm{ME} / \mathrm{TB}$ mixtures, respectively. To guide the eye the data were fitted by a polynomial of the third degree. The analogous changes at different wavenumbers look more or less similar. The largest deviations for ME/ET mixture occur near $X_{\mathrm{ET}} \approx 0.5-0.55$. In the case of $\mathrm{ME} / \mathrm{TB}$ mixture this value shifts to $X_{\mathrm{TB}} \approx 0.55-0.6$ (Fig. 12). This small shift from the equimolar composition may results from formation of TB-rich clusters, in addition to the $1: 1$ clusters. Sengawa et al. reported that the maximum negative values of the excess static dielectric constant for ME/ET mixture were observed at mole fraction of $X \approx 0.5 .{ }^{24}$ Hence, the authors concluded that the interaction between methanol and ethanol is the strongest at equimolar mixture. Since the excess static dielectric constant was negative in the entire range of compositions it was suggested that the number of hydrogen bonds in the mixture is fewer than for the corresponding pure liquids. ${ }^{24} \mathrm{~A}$ careful analysis of Fig. 10 led us to quite different conclusions. The negative values of the excess MIR $\left(3200-3600 \mathrm{~cm}^{-1}\right)$ and NIR (6200-7000 $\mathrm{cm}^{-1}$ ) absorption of the bonded $\mathrm{OH}$ reveal that an average population and/or strength of hydrogen bonds decreases on going from the ideal to the real mixture. Yet, in this case we should observe an opposite trend for the free $\mathrm{OH}$. It is of particular note that the peaks due to the free $\mathrm{OH}$ are negative as well. This effect is well seen for ME/TB mixture (Fig. 10b, red line), where one can observe a distinct negative

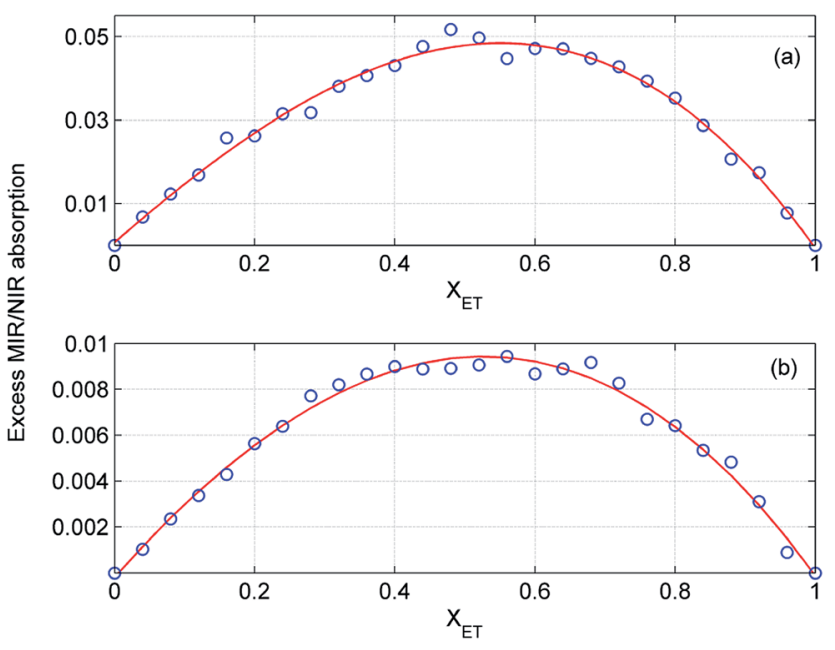

Fig. 11 Intensity changes at $2978 \mathrm{~cm}^{-1}$ (a) and $8440 \mathrm{~cm}^{-1}$ (b) in the excess absorption spectra of ME/ET mixture. The experimental data $(O)$ were fitted by a polynomial of the third degree (red line). 


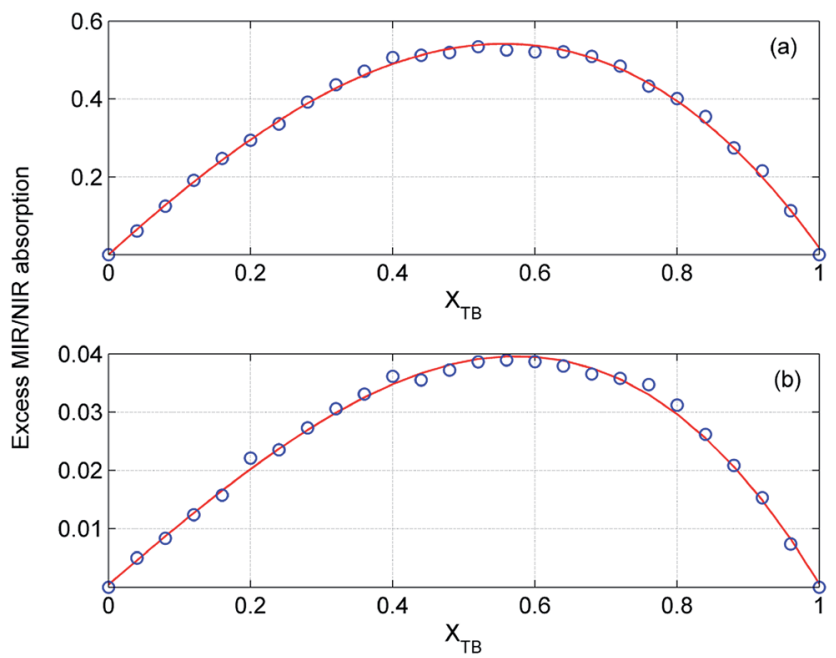

Fig. 12 Intensity changes at $1199 \mathrm{~cm}^{-1}$ (a) and $8432 \mathrm{~cm}^{-1}$ (b) in the excess absorption spectra of ME/TB mixture. The experimental data (O) were fitted by a polynomial of the third degree (red line).

peak at $7060 \mathrm{~cm}^{-1}$ from the free $\mathrm{OH}$ of TB. These observations allow us to infer that in the mixture increases the population of hydrogen-bonded $\mathrm{OH}$ groups as compared with the pure alcohols, but an average strength of these bonds is weaker. The formation of heteroclusters leads to significant reduction in the population of the free $\mathrm{OH}$ groups from TB (Fig. 10b). On the other hand, participation of molecules of $\mathrm{ME}$ in the mixed clusters decreases an average strength of hydrogen bonds since the ME-TB bonds are weaker than those of ME-ME.

\section{Theoretical modeling of structure and stability of homo- and heteroclusters}

Fig. 13 shows the theoretical energies of the mixed ME/TB cyclic tetramers (a), pentamers (b) and hexamers (c) as a function of the cluster composition. We focuses on these kinds of clusters since there are numerous reports showing that the cyclic tetramers, pentamers and hexamers are the most abundant species in the liquid alcohols. ${ }^{25-31}$ As expected, an increase in the number of TB molecules in the cluster increases the energy of the cluster as the most favorable ME-ME hydrogen bonds are replaced by ME-TB bonds. On the other hand, it is evident that the energy of the mixed clusters is smaller than that resulting from the sum of contributions from the homoclusters (straight line). The straight line in Fig. 13 represents an ideal mixture. It is of particular note that the largest deviation from the ideality is observed for clusters with the equimolar composition. This effect is more clearly seen for even-numbered clusters (tetramers, hexamers). Hence, we have the theoretical confirmation of the results obtained from the excess absorption spectra (Fig. 11). As shown, for ME/TB mixture this point is shifted to higher values of $X_{\mathrm{TB}}$ (Fig. 12) as a result of the presence of two kinds of mixed clusters. On the other hand, the relationships shown in Fig. 13 are based on modeling of one kind of clusters.

The theoretical calculations show that formation of the mixed clusters decreases an average energy as compared with
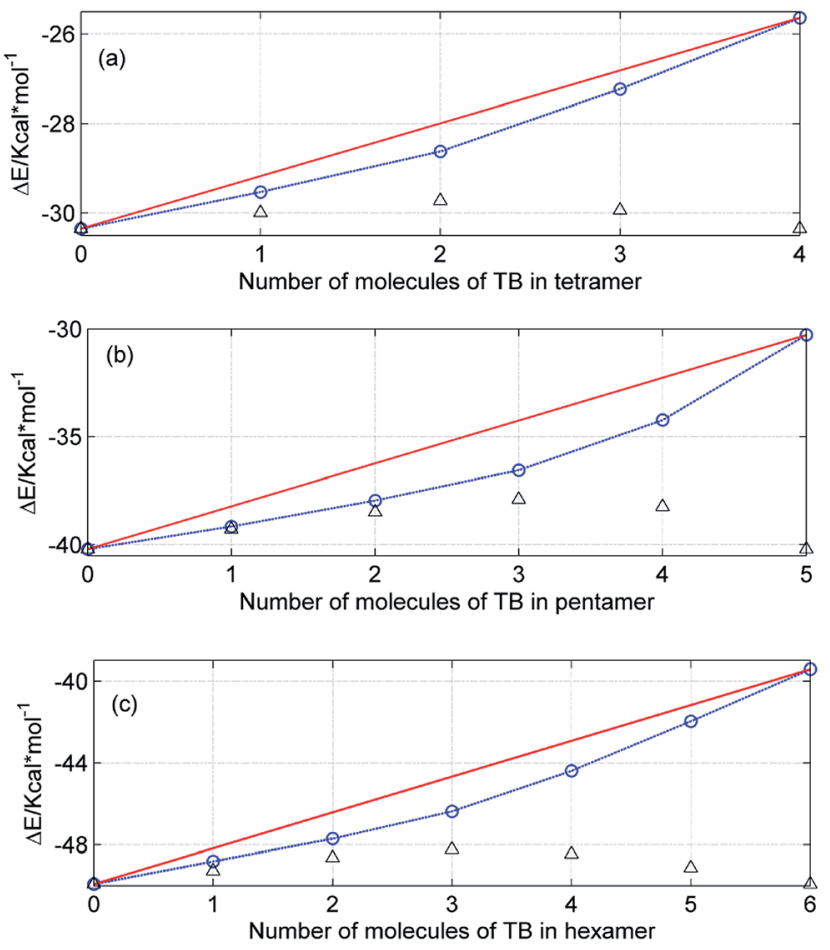

Fig. 13 Theoretical energies (DFT) of the mixed ME/TB tetramers (a), pentamers (b) and hexamers (c) as a function of the cluster composition. The red line represents an ideal mixture, while the triangles show the deviation from the ideality (the symbols were shifted to appear in this scale).

an ideal cluster. In other words, an average strength of hydrogen bonds in the real mixture is expected to be higher than that in the ideal mixture. On the other hand, from the excess absorption spectra (Fig. 10) it results that an average strength of hydrogen bonds decreases on going from pure alcohols to the mixture. The creation of the mixed clusters from the hydrogenbonded components only should decrease the energy as shown in Fig. 13. Yet, we have shown that creation of the mixed clusters involves largely molecules of TB with the free $\mathrm{OH}$ groups. This way upon mixing of ME and TB increases the overall number of hydrogen bonds but an average strength of these bonds is smaller as compared with the ideal mixture.

\section{Conclusions}

Chemometric and 2D correlation analysis reveal the presence of three kinds of clusters in most of the studied mixtures: two kinds of homoclusters and one kind of heterocluster. The composition of this mixed cluster depends on the composition of the mixture but an average molar ratio is close to $1: 1$. Only for ME/TB mixture, in addition to these three clusters, TB-rich cluster with an average ME : TB molar ratio between $1: 3$ and $1: 4$ was found. Our results evidence that the extent of nonideality in the binary mixtures of ME with the short chain aliphatic alcohol depends on the chain length and the order of alcohol. It is of particular note that this tendency is perfectly correlated with the degree of the self-association of the alcohol. 
As a result, behavior of ME/ET mixture is the closest to the ideal mixture, while the largest deviation from the ideality occurs for ME/TB mixture. From the MIR/NIR excess absorbance spectra it results that the largest magnitude of these changes is observed at mole fraction of $X \approx 0.5$. Only for $\mathrm{ME} / \mathrm{TB}$ mixture this point is shifted to $X_{\mathrm{TB}} \approx 0.55-0.6$. For all studied mixtures at equimolar composition about 50\% molecules are involved in the heteroclusters, while the remaining molecules form the homoclusters similar to those found in pure alcohols. Results of MCR-ALS indicate that in spite of different chain length and structure all the mixtures show similar behavior. On this basis we can conclude that the structure of the mixtures of ME with short chain alcohols is determined mainly by the $\mathrm{OH}-\mathrm{OH}$ interactions, while the chain length and structure have a less important meaning. On the other hand, one can expect that increasing chain length of both components of the mixture may affect the extent of the separation more appreciably. Such studies are in progress and will be reported elsewhere.

Our results confirmed the presence of heterogeneity at molecular level in ME/ET mixture, as reported by Mello et al. ${ }^{12}$ However, in addition to the homoclusters of ME and ET we observed also significant amount of heteroclusters in the entire range of compositions. It does not seem likely that similar molecules of ME and ET do not form the mixed clusters, whereas more unlike molecules of $\mathrm{ME}$ and acetone are able to from the heteroclusters. ${ }^{7-9}$ Besides, the presence of pure ME and ET clusters only would imply that the strength of hydrogen bonding is a linear function of the composition of the mixture. In this case, the asynchronous spectrum should not develop any significant peaks. ${ }^{15-17}$

\section{Acknowledgements}

This work was supported by Grant NCN 2013/11/B/ST4/00501.

\section{References}

1 S. Dixit, J. Crain, W. C. K. Poon, J. L. Finney and A. K. Soper, Nature, 2002, 416, 829.

2 A. K. Soper, L. Dougan, J. Crain and J. L. Finney, J. Phys. Chem. B, 2006, 110, 3472.

3 A. Wakisaka, K. Matsuura, M. Uranaga, T. Sekimoto and M. Takahashi, J. Mol. Liq., 2011, 160, 103.

4 A. Wakisaka and T. Ohki, J. Mol. Liq., 2005, 129, 231.

5 G. Onori, Chem. Phys. Lett., 1989, 154, 212.

6 K. Yoshida and T. Yamaguchi, Z. Naturforsch., A: Phys. Sci., 2001, 56, 529.

7 M. Musso, M. G. Giorgini and H. Torii, J. Mol. Liq., 2008, 147, 37.

8 A. Idrissi, K. Polok, B. Marekha, I. Dewaele, M. Bria and W. Gadomski, J. Phys. Chem. B, 2014, 118, 1416.

9 F. Kollipost, A. V. Domanskaya and M. A. Shum, J. Phys. Chem. A, 2015, 119, 2225.

10 N. Y. Tan, R. Li, P. Bräuer, C. D'Agostino and L. F. Gladden, Phys. Chem. Chem. Phys., 2015, 17, 5999.

11 J. Ortega, C. Ràfols, E. Bosch and M. Rosés, J. Chem. Soc., Perkin Trans. 2, 1996, 1497.
12 C. Mello, T. Mello, E. Sevéri, L. Coelho, D. Ribeiro, A. Marangoni, R. J. Poppi and I. Noda, J. Chem. Phys., 2009, 131, 084501.

13 Q. Li, N. Wang, Q. Zhou, S. Sun and Z. Yu, Appl. Spectrosc., 2008, 62, 166.

14 Y. Koga, F. Sebe, T. Minami, K. Otake, K. Saitow and K. Nishikawa, J. Phys. Chem. B, 2009, 113, 11928.

15 I. Noda, Appl. Spectrosc., 1993, 47, 1329.

16 I. Noda and Y. Ozaki, Two Dimensional Correlation Spectroscopy-Applications in Vibrational and Optical Spectroscopy, John Wiley \& Sons, Ltd., England, 2004.

17 M. A. Czarnecki, Appl. Spectrosc. Rev., 2011, 46, 67.

18 S. Wold, K. Esbensen and P. Geladi, Chemom. Intell. Lab. Syst., 1987, 2, 37.

19 H. R. Keller and D. L. Massart, Chemom. Intell. Lab. Syst., 1991, 12, 209.

20 R. Tauler, B. Kowalski and S. Fleming, Anal. Chem., 1993, 65, 2040.

21 R. Tauler, A. Izquierdo-Ridorsa and E. Casassas, Chemom. Intell. Lab. Syst., 1993, 18, 293.

22 M. J. Frisch, G. W. Trucks, H. B. Schlegel, G. E. Scuseria, M. A. Robb, J. R. Cheeseman, J. A. Montgomery Jr, T. Vreven, K. N. Kudin, J. C. Burant, J. M. Millam, S. S. Iyengar, J. Tomasi, V. Barone, B. Mennucci, M. Cossi, G. Scalmani, N. Rega, G. A. Petersson, H. Nakatsuji, M. Hada, M. Ehara, K. Toyota, R. Fukuda, J. Hasegawa, M. Ishida, T. Nakajima, Y. Honda, O. Kitao, H. Nakai, M. Klene, X. Li, J. E. Knox, H. P. Hratchian, J. B. Cross, C. Adamo, J. Jaramillo, R. Gomperts, R. E. Stratmann, O. Yazyev, A. J. Austin, R. Cammi, C. Pomelli, J. W. Ochterski, P. Y. Ayala, K. Morokuma, G. A. Voth, P. Salvador, J. J. Dannenberg, V. G. Zakrzewski, S. Dapprich, A. D. Daniels, M. C. Strain, O. Farkas, D. K. Malick, A. D. Rabuck, K. Raghavachari, J. B. Foresman, J. V. Ortiz, Q. Cui, A. G. Baboul, S. Clifford, J. Cioslowski, B. B. Stefanov, G. Liu, A. Liashenko, P. Piskorz, I. Komaromi, R. L. Martin, D. J. Fox, T. Keith, M. A. Al-Laham, C. Y. Peng, A. Nanayakkara, M. Challacombe, P. M. W. Gill, B. Johnson, W. Chen, M. W. Wong, C. Gonzalez and J. A. Pople, Gaussian 03, Revision C. 02, Gaussian, Inc, Wallingford CT, 2004.

23 M. A. Czarnecki, Y. Morisawa, Y. Futami and Y. Ozaki, Chem. Rev., 2015, 115, 9707.

24 R. J. Sengawa, S. Sankhla and N. Shinyashiki, J. Solution Chem., 2008, 37, 137.

25 A. N. Fletcher, J. Phys. Chem., 1969, 73, 2217.

26 S. Sarkar and R. N. Joarder, J. Chem. Phys., 1993, 99, 2032.

27 G. M. Førland, F. O. Libnau, O. M. Kvalheim and H. Høiland, Appl. Spectrosc., 1996, 50, 1264.

28 L. Stordrange, A. A. Christy, O. M. Kvalheim, H. Shen and Y. Z. Liang, J. Phys. Chem. A, 2002, 106, 8543.

29 R. Ludwig, T. Weinhold and T. C. Farrar, Mol. Phys., 1999, 97, 465.

30 R. Ludwig, T. Weinhold and T. C. Farrar, Mol. Phys., 1999, 97, 479.

31 M. Huelsekopf and R. Ludwig, J. Mol. Liq., 2000, 85, 105. 